\title{
Auxin regulates anthocyanin biosynthesis through the Aux/IAA-ARF signaling pathway in apple
}

Yi-cheng Wang ${ }^{1,2}$, Nan Wang ${ }^{1,2}$, Hai-feng X $u^{1,2}$, Sheng-hui Jiang ${ }^{1,2}$, Hong-cheng Fang ${ }^{1,2}$, Meng-yu Su ${ }^{1,2}$, Zong-ying Zhang ${ }^{1,2}$, Tian-liang Zhang ${ }^{1,2}$ and Xue-sen Chen ${ }^{1,2}$

\begin{abstract}
Auxin signaling, which is crucial for normal plant growth and development, mainly depends on ARF-Aux/IAA interactions. However, little is known regarding the regulatory effects of auxin signaling on anthocyanin metabolism in apple (Malus domestica). We investigated the functions of MdARF13, which contains a repression domain and is localized to the nucleus. This protein was observed to interact with the Aux/IAA repressor, MdIAA121, through its Cterminal dimerization domain. Protein degradation experiments proved that MdIAA121 is an unstable protein that is degraded by the 265 proteasome. Additionally, MdIAA121 stability is affected by the application of exogenous auxin. Furthermore, the overexpression of MdIAA121 and MdARF13 in transgenic red-fleshed apple calli weakened the inhibitory effect of MdARF13 on anthocyanin biosynthesis. These results indicate that the degradation of MdIAA121 induced by auxin treatment can release MdARF13, which acts as a negative regulator of the anthocyanin metabolic pathway. Additionally, yeast two-hybrid, bimolecular fluorescence complementation, and pull-down assays confirmed that MdMYB10 interacts with MdARF13. A subsequent electrophoretic mobility shift assay and yeast one-hybrid assay demonstrated that MdARF13 directly binds to the promoter of MdDFR, which is an anthocyanin pathway structural gene. Interestingly, chromatin immunoprecipitation-quantitative real-time PCR results indicated that the overexpression of MdIAA121 clearly inhibits the recruitment of MdARF13 to the MdDFR promoter. Our findings further characterized the mechanism underlying the regulation of anthocyanin biosynthesis via Aux/IAA-ARF signaling.
\end{abstract}

\section{Introduction}

Color is an important fruit quality index that influences the purchasing behavior of consumers to some degree ${ }^{1}$. As a type of secondary metabolite, anthocyanins constitute one of the main pigments responsible for fruit color $^{2,3}$. Additionally, their positive effects on human health include enhancing vascular elasticity, preventing cardiovascular disease, and protecting the liver from damage $e^{4-7}$.

Correspondence: X.-s. Chen (chanyetixi@163.com)

${ }^{1}$ State Key Laboratory of Crop Biology, College of Horticulture Science and Engineering, Shandong Agricultural University, Tai-An, Shandong, China ${ }^{2}$ Collaborative Innovation Center of Fruit \& Vegetable Quality and Efficient Production in Shandong, Tai-An, Shandong, China
Three main protein families (MYB, bHLH, and WD40) regulate anthocyanin biosynthesis by forming the MYB-bHLH-WD40 complex ${ }^{8,9}$. The MYB transcription factors (TFs) belong to one of the largest plant TF families, with some members associated with anthocyanin biosynthesis having been isolated and identified in many plant species, including Arabidopsis thaliana, maize, strawberry, and grape ${ }^{10-13}$. In apple, the three genes encoding MYB activators (MYB10, MYB1, and MYBA) involved in anthocyanin biosynthesis are alleles $^{14}$. Two MYB TF genes (MdMYB1 and MdMYBA), which were first isolated from fruit skin, have been confirmed to be responsible for the accumulation of anthocyanin $^{15,16}$. Furthermore, $M d M Y B 10$ consists of a characteristic R6 rearrangement in its promoter that is

\section{(c) The Author(s) 2018}

(c) (i) Open Access This article is licensed under a Creative Commons Attribution 4.0 International License, which permits use, sharing, adaptation, distribution and reproduction in any medium or format, as long as you give appropriate credit to the original author(s) and the source, provide a link to the Creative Commons license, and indicate if changes were made. The images or other third party material in this article are included in the article's Creative Commons license, unless indicated otherwise in a credit line to the material. If material is not included in the article's Creative Commons license and your intended use is not permitted by statutory regulation or exceeds the permitted use, you will need to obtain permission directly from the copyright holder. To view a copy of this license, visit http://creativecommons.org/licenses/by/4.0/. 
associated with increased anthocyanin biosynthesis in red-fleshed apples ${ }^{17}$.

Anthocyanin biosynthesis is influenced by environmental stimuli (e.g., light and temperature) and plant hormones (e.g., auxin and jasmonate $)^{18-21}$. Moreover, auxins (naphthalene acetic acid (NAA) or 2,4-dichlorophenoxyacetic acid (2,4-D)) regulate secondary metabolic pathways, including those related to phenylpropanoid, flavonoid, and anthocyanin metabolism ${ }^{22-25}$. An earlier study involving a carrot suspension culture revealed that 2,4-D strongly inhibits anthocyanin biosynthesis ${ }^{20}$. Additionally, the application of exogenous NAA and 2,4-D can decrease the anthocyanin contents of transgenic tobacco calli ${ }^{24}$. In apple, studies involving red-fleshed apple calli revealed that increasing auxin concentrations can significantly inhibit anthocyanin biosynthesis ${ }^{26,27}$. However, the role of the auxin signaling pathway during the regulation of anthocyanin biosynthesis is unclear.

Auxin (indole-3-acetic acid (IAA)) regulates plant development by inducing rapid cellular responses and changes in gene expression. Auxin response factors (ARFs) together with auxin/IAA (Aux/IAA) proteins are TFs that function as key regulators of auxin-responsive transcription in plants ${ }^{28-30}$. Current auxin signaling models indicate that auxin responses mainly depend on the interaction between the homologous C-terminal domains of Aux/IAA and ARF proteins ${ }^{31,32}$. When cellular auxin concentrations are low, ARF activators at the promoters of auxin-responsive genes are thought to be inactive because of their association with Aux/IAA repressors ${ }^{29,31}$. When auxin concentrations increase, the Aux/IAA repressors are recruited to auxin receptors and degraded via the ubiquitin-proteasome pathway ${ }^{33,34}$. This degradation enables ARF activators to repress/activate the expression of auxin-responsive genes ${ }^{33}$.

Most ARFs consist of an N-terminal DNA-binding domain (DBD), a middle region that functions as an activation domain (AD) or a repression domain (RD), and a C-terminal dimerization domain $(\mathrm{CTD})^{35}$. ARFs can specifically bind to the TGTCTC auxin-response element (AuxRE) in the promoters of primary/early auxinresponse genes ${ }^{35}$. In the last few years, several new details regarding the role of ARFs in plant growth and development have been revealed. For example, AtARF3 and AtARF4 are important for the development of reproductive and vegetative tissues ${ }^{36,37}$. Additionally, AtARF5 is involved in embryonic and vascular tissue development ${ }^{38,39}$, while OsARF8 mediates hypocotyl elongation and influences auxin homeostasis ${ }^{40}$. Meanwhile, SlARF7 may negatively regulate fruit set, whereas the upregulated expression of SIARF10 suggests that the encoded protein positively affects fruit development ${ }^{41,42}$. Thus, ARFs exhibit dynamic functions in plants and have a central role in the hub of transcriptional networks.
In the present study, we investigated the effects of different auxin concentrations on anthocyanin biosynthesis in red-fleshed apple calli. We also characterized an $A R F$ gene, MdARF13, and confirmed that the MdARF13mediated auxin signaling pathway helps regulate anthocyanin biosynthesis in apple, thereby revealing a novel metabolic function for ARF proteins.

\section{Materials and methods}

\section{Plant materials and hormone treatments}

Calli induced as previously described were cultured on Murashige and Skoog (MS) medium supplemented with $2 \mu \mathrm{mol} / \mathrm{L}$ 6-benzylaminopurine and 5, 10, 20, or $40 \mu \mathrm{mol} /$ $\mathrm{L} \mathrm{NAA}^{43}$. The $\mathrm{pH}$ of all media was adjusted to $5.8 \pm 0.1$. The red-fleshed apple calli were incubated under a $16 \mathrm{~h}$ light $/ 8 \mathrm{~h}$ dark photoperiod (light intensity: 1000-2000 lx) for 18 days before being harvested.

\section{Anthocyanin extraction and absorbance measurements}

Harvested calli $(0.5 \mathrm{~g}$ each) were ground to a powder in liquid nitrogen and then treated with $15 \mathrm{~mL} \mathrm{1 \%} \mathrm{(v/v)} \mathrm{HCl}$ methanol for $24 \mathrm{~h}$ at $4{ }^{\circ} \mathrm{C}$ in darkness. The solutions were then centrifuged for $10 \mathrm{~min}$ at $8000 \times g$. The absorbance of the supernatants was measured at $530 \mathrm{~nm}$ using a UV2450 spectrophotometer (Shimadzu, Kyoto, Japan).

\section{RNA isolation and quantitative real-time polymerase chain reaction}

Total RNA was isolated using an RNAprep Pure Plant Kit (Tiangen, Beijing, China). First-strand complementary DNA (cDNA) was synthesized using the RevertAid FirstStrand cDNA Synthesis Kit (Fermentas, St. Leon-Roth, Germany). Three replicates were prepared for each sample. Quantitative real-time polymerase chain reaction (qPCR) analyses were conducted using $50 \mathrm{ng} / \mu \mathrm{L}$ cDNA as the template, SYBR Green PCR Master Mix (TransGen Biotech, Beijing, China), and the iCycler iQ5 system (BioRad, Hercules, CA, USA). Details regarding the qPCR primers are provided in Supplementary Table S1. MdActin was used as the internal control.

\section{Amino acid sequence analysis and phylogenetic tree construction}

The ARF amino acid sequences were obtained from the NCBI (National Center for Biotechnology Information) database and aligned using the DNAMAN software (Lynnon Biosoft, USA). A phylogenetic tree was constructed according to the neighbor-joining method using the MEGA5.0 program.

\section{Red-fleshed apple callus transformation}

The intact MdARF13 coding sequence (CDS) was inserted into the pRI101 vector containing the $35 \mathrm{~S}$ promoter and a green fluorescent protein (GFP) tag sequence to generate 
a
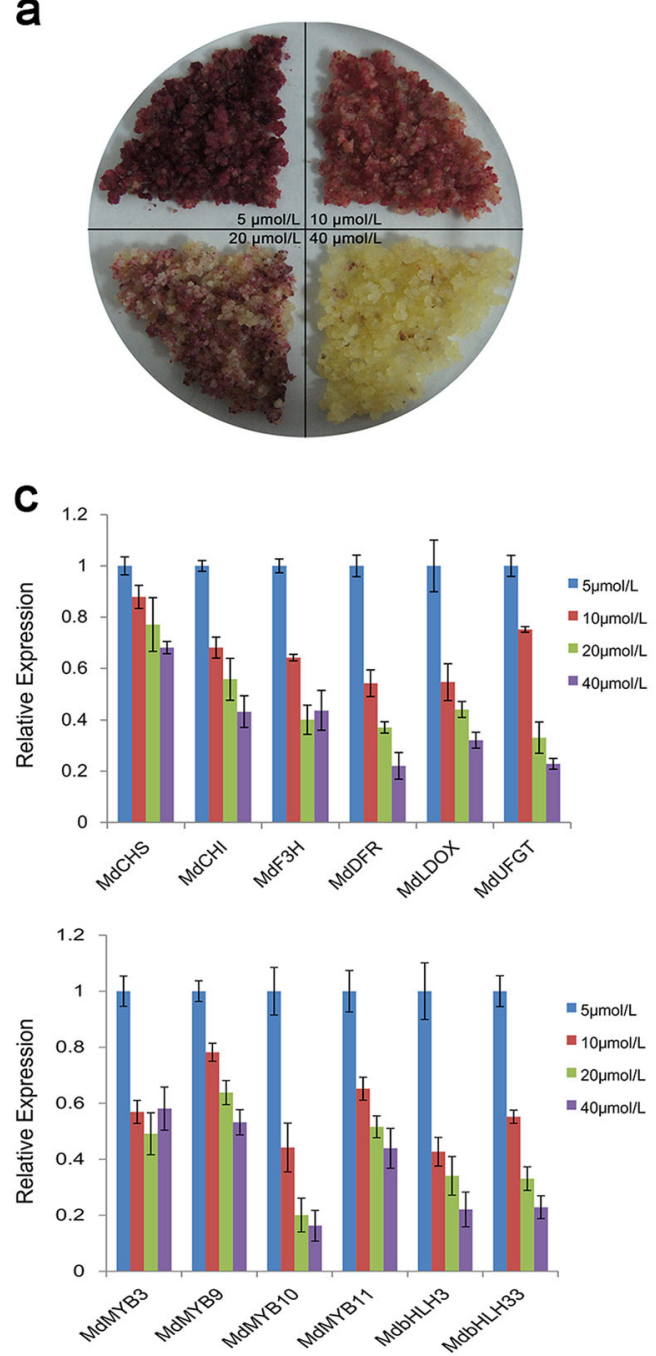

b
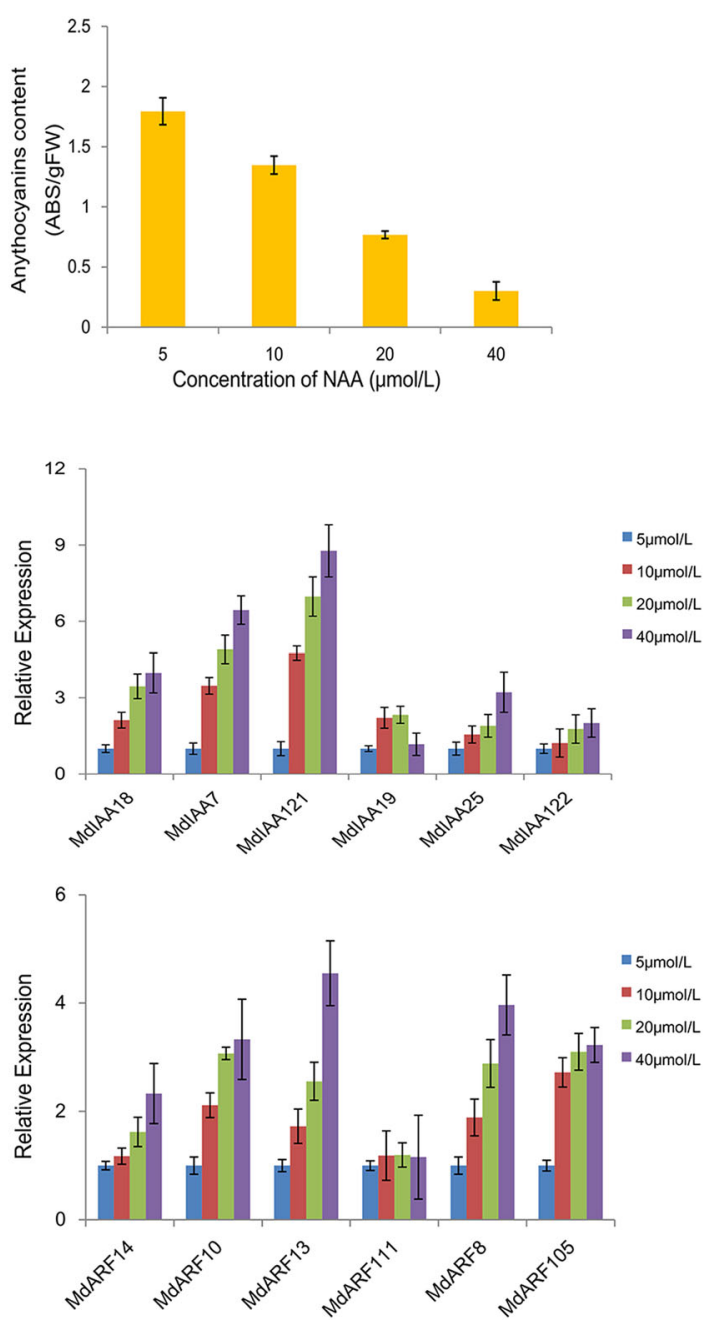

Fig. 1 Red-fleshed apple calli on medium supplemented with different NAA concentrations $(\mathbf{5}, \mathbf{1 0}$, 20, and 40 mmol/L). a Phenotypes of calli treated with different NAA concentrations, as well as the extraction of anthocyanin. $\mathbf{b}$ Relative anthocyanin content at 18 days. The relative anthocyanin content was calculated as follows: absorbance $(530 \mathrm{~nm}) /$ fresh weight $(\mathrm{g})$. c Relative expression levels of anthocyanin biosynthesis-related genes in response to different NAA treatments at 18 days

the 35S::MdARF13-GFP construct. Additionally, the intact MdIAA121 CDS was inserted into the pCAMBIA1301 vector containing the $35 \mathrm{~S}$ promoter and a GFP tag sequence to prepare the 35S::MdIAA121-GFP construct. The resulting recombinant vectors were transferred to Agrobacterium tumefaciens LBA4404 cells. Two-week-old calli grown in liquid medium were cocultured with $A$. tumefaciens LBA4404 cells carrying the 35S::MdARF13GFP construct on MS medium containing $2 \mu \mathrm{mol} / \mathrm{L}$ NAA and $4 \mu \mathrm{mol} / \mathrm{L} 6$-benzylaminopurine at $25^{\circ} \mathrm{C}$ for 2 days in darkness. The calli were then transferred to fresh MS medium supplemented with $662 \mu \mathrm{mol} / \mathrm{L}$ carbenicillin and $74 \mu \mathrm{mol} / \mathrm{L}$ kanamycin to screen for transgene-carrying calli. To obtain calli cotransfected with MdARF13+MdIAA121, the same method was used, but calli were transferred to MS medium supplemented with $662 \mu \mathrm{mol} / \mathrm{L}$ carbenicillin, $74 \mu \mathrm{mol} / \mathrm{L}$ kanamycin, and $40 \mu \mathrm{mol} / \mathrm{L}$ hygromycin. GFPoverexpressing calli were used as controls.

\section{Yeast two-hybrid assay}

Yeast (Saccharomyces cerevisiae) two-hybrid ( $\mathrm{Y} 2 \mathrm{H})$ assays were conducted according to the manufacturer's instructions (Clontech, Mountain View, CA, USA). The MdARF13 CDS was inserted into the pGBKT7 vector (i.e., bait), while the MdIAA7, MdIAA121, MdMYB9, and MdMYB10 CDSs were inserted into the pGADT7 vector (i.e., prey). All recombinant vectors were cotransformed into yeast strain Y2H Gold cells using the lithium acetate method. The cells 

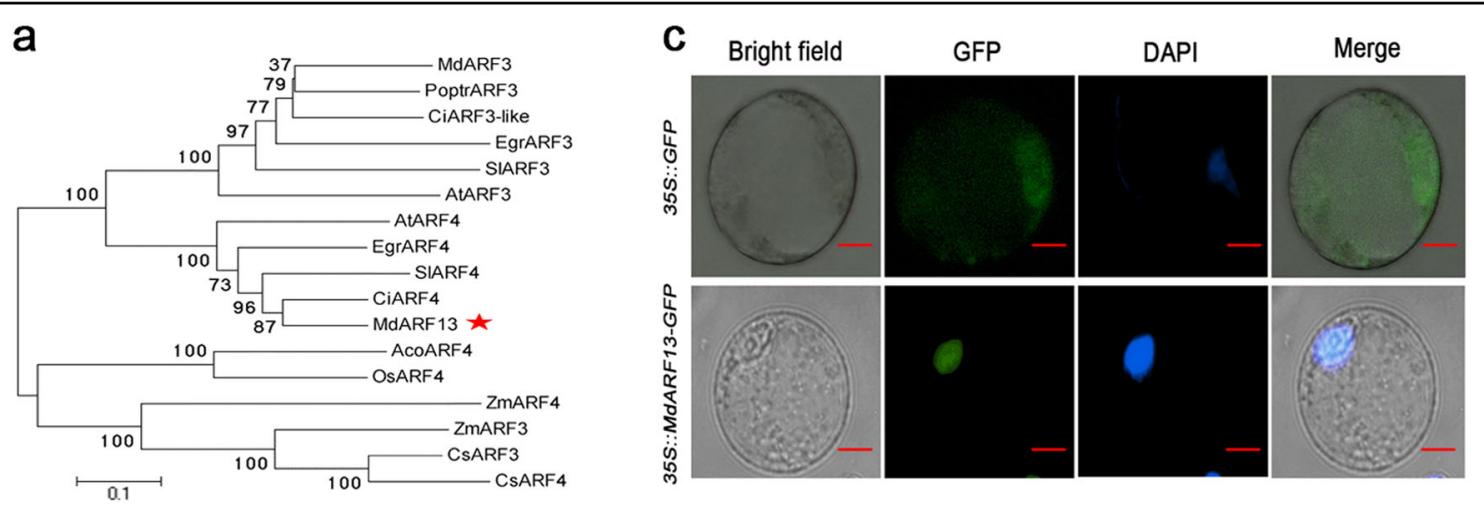

b

B3-like DNA Binding Domain

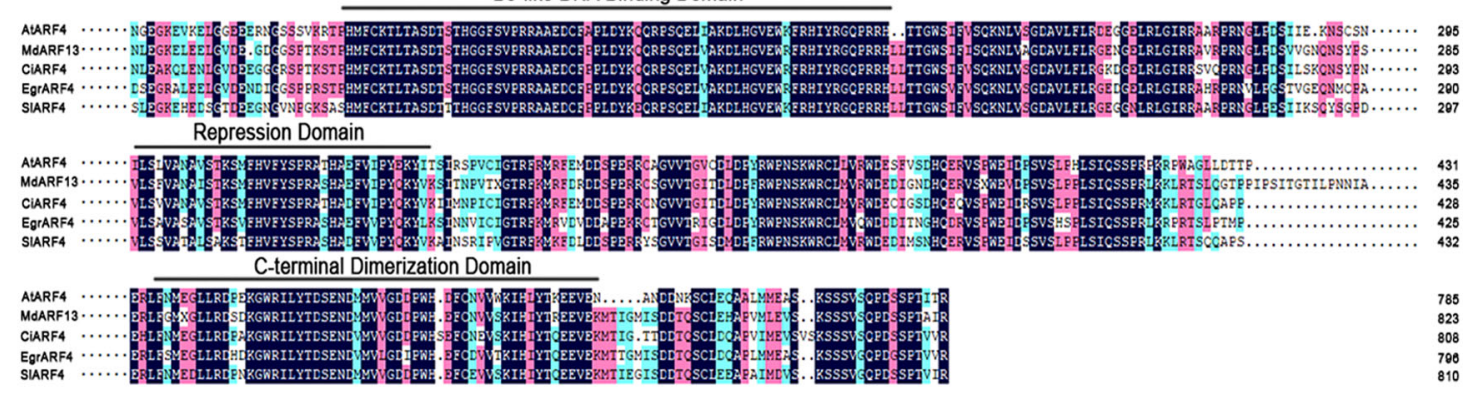

Fig. 2 Bioinformatic analyses and MdARF13 subcellular localization. a Phylogenetic analysis of ARF transcription factors in different species. b Alignment of MdARF13, AtARF4, CiARF4, EgrARF4, and SiARF4 proteins. c Subcellular localization of the MdARF13-GFP fusion protein in transformed apple callus protoplasts. The protoplasts producing GFP alone were used as the control. Bars $=50 \mu \mathrm{m}$

were cultured on synthetic defined (SD) medium lacking leucine and tryptophan (SD/-Leu/-Trp). Putative transformants were transferred to SD medium lacking adenine, histidine, leucine, and tryptophane (SD/-Ade/-His/-Leu/ -Trp; Clontech) with or without X- $\alpha$-gal.

\section{Bimolecular fluorescence complementation assay}

For a bimolecular fluorescence complementation (BiFC) assay, the MdARF13 CDS was inserted into the pSPYNE-35S vector (with an NYFP tag sequence) to generate the MdARF13-NYFP recombinant vector. The MdIAA121 and MdMYB10 CDSs were inserted into the pSPYCE-35S vector (with a CYFP tag sequence) to generate the MdIAA121-CYFP and MdMYB10-CYFP recombinant vectors. A. tumefaciens LBA4404 cells were transformed with the recombinant vectors and then cultured in liquid medium until the optical density $(600 \mathrm{~nm})$ reached 0.6. Onion epidermal cells were treated with equal volumes of different combinations of $A$. tumefaciens strains. Onion cells were cultured at $23^{\circ} \mathrm{C}$ for $48 \mathrm{~h}$. The yellow fluorescence protein (YFP) signal was detected using a Zeiss CLSM-5 confocal laser scanning microscope (excitation wavelength of $488 \mathrm{~nm}$ ).

\section{Pull-down assay}

The MdIAA121 and MdMYB10 open reading frames were cloned into the pET32a vector containing a poly-histidine
(HIS) tag sequence. The intact MdARF13 CDS was ligated into the pGEX-4T-1 vector containing a glutathione $S$ transferase (GST) tag sequence. The recombinant vectors were inserted into Escherichia coli BL21 (DE3) cells (TransGen) to induce the production of fusion proteins. The resulting proteins were mixed together and then purified in columns using the HIS tag. The purified mixed proteins were subsequently analyzed by western blotting with antiHIS or anti-GST antibodies (Abmart, Shanghai, China).

\section{Yeast one-hybrid analysis}

Yeast one-hybrid $(\mathrm{Y} 1 \mathrm{H})$ assays were conducted using yeast strain Y187 cells (Clontech) according to the manufacturer's instructions. MdARF13 was cloned into the pGADT7 vector, while the $M d D F R$ promoter was inserted into the pHIS2 vector. Different combinations of recombinant and empty vectors were cotransformed into yeast Y187 cells, and the interactions were examined on SD medium lacking Trp, Leu, and His (SD/-Trp/-Leu/-His) with an optimal concentration of 3-amino-1,2,4-triazole.

\section{Electrophoretic mobility shift assays}

Electrophoretic mobility shift assay (EMSA) experiments were completed using an EMSA kit (Pierce, Rockford, IL, USA) and biotin-labeled probes. Briefly, biotin-labeled probes were incubated in $1 \times$ binding buffer 


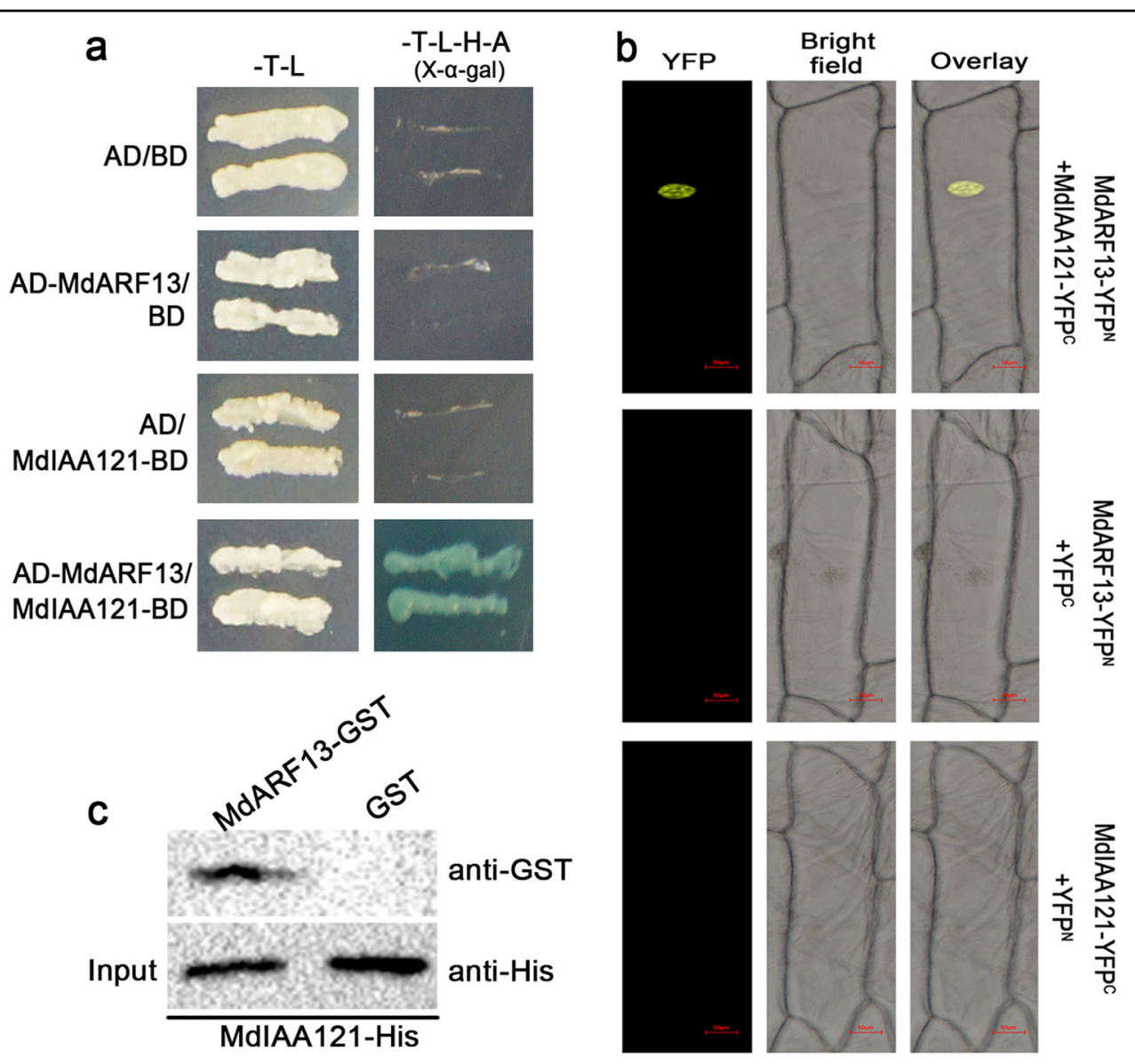

Fig. 3 MdARF13 is targeted by MdIAA121. The interaction between MdARF13 and MdlAA121 was verified in a a yeast two-hybrid assay, $\mathbf{b}$ a bimolecular fluorescence complementation assay, and $\mathbf{c}$ a pull-down assay

(2.5\% glycerol, $10 \mathrm{mM}$ EDTA, $5 \mathrm{mM} \mathrm{MgCl}_{2}$, and $50 \mathrm{mM}$ $\mathrm{KCl}$ ) with or without proteins at $24{ }^{\circ} \mathrm{C}$ for $25 \mathrm{~min}$. An unlabeled probe was added to the reactions.

\section{Chromatin immunoprecipitation-quantitative real-time PCR analysis}

The chromatin immunoprecipitation (ChIP) experiment was completed using a modified version of a published method $^{44}$. The ChIP Assay Kit (Upstate Biotechnology, Lake Placid, NY, USA) and anti-GFP antibody (Abmart) were used for cross-linking, the removal of cross-linkers, immunoprecipitation, and elution. The ChIP signal was quantified by qPCR as the percentage of the total input DNA. The experiment was repeated three times.

\section{Subcellular localization analysis}

Protoplasts isolated from apple callus cells were prepared and transformed as described by $\mathrm{Hu}$ et $\mathrm{al}^{45}$. Transformed apple protoplasts were stained with the DNA-specific dye 4',6-diamidino-2-phenylindole, which highlights the location of the nucleus. The protoplasts from transgenic calli were observed by fluorescence microscopy.

\section{In vitro protein degradation assay}

Protein degradation assays were used to detect the dynamic changes to MdIAA13 in vitro. The assay buffer contained $25 \mathrm{mM}$ Tris- $\mathrm{HCl}, \mathrm{pH} 7.5,10 \mathrm{mM} \mathrm{NaCl}, 10 \mathrm{mM}$ $\mathrm{MgCl}_{2}, 5 \mathrm{mM}$ dithiothreitol, $10 \mathrm{mM} \mathrm{ATP}$, and $4 \mathrm{mM}$ phenylmethylsulfonyl fluoride. Wild-type red-fleshed calli were treated with buffer, and the extract was incubated with the MdIAA13-His fusion protein for specific periods. The relative MdIAA121 abundance was detected by western blot using anti-His monoclonal antibodies.

\section{Results}

Anthocyanin content and related gene expression under different auxin concentrations

The anthocyanin contents of red-fleshed apple calli gradually decreased with increasing NAA concentrations (5-40 $\mu \mathrm{mol} / \mathrm{L})$, which caused the callus color to fade (Fig. 1a). Moreover, the anthocyanin content of calli cultured in $5 \mu \mathrm{mol} / \mathrm{L}$ NAA was 1.39-, 2.42-, and 5.98-fold higher than that of calli grown in 10,20 , and $40 \mu \mathrm{mol} / \mathrm{L}$ NAA, respectively (Fig. 1b). These results are consistent with the findings of Ji et al. ${ }^{26,27}$ and implied that low auxin 


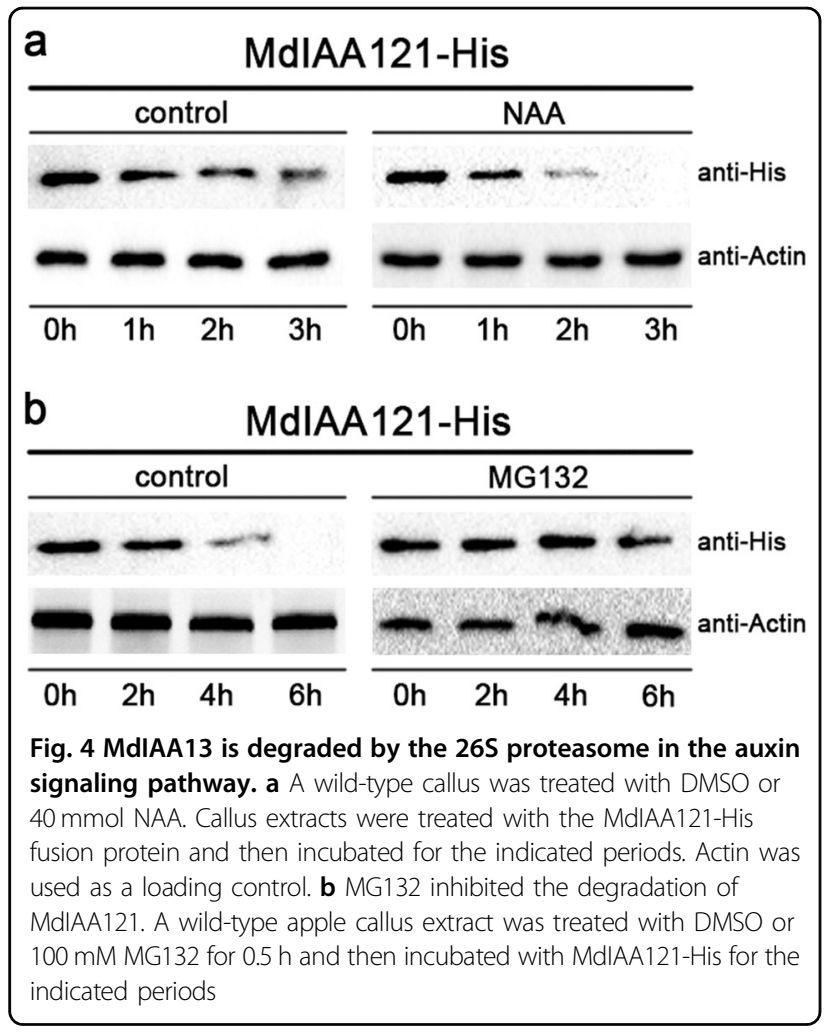

concentrations were conducive to anthocyanin accumulation in red-fleshed apple calli.

The changes in the anthocyanin content of calli grown under different auxin conditions were also reflected at the transcript level. As the auxin concentration increased, the expression levels of anthocyanin biosynthesis-related genes decreased by varying degrees (Fig. 1c). However, the transcript levels for the $A u x / I A A$ and $A R F$ genes increased with increasing NAA concentrations (Fig. 1c). In particular, the MdARF13 expression levels in calli treated with $40 \mu \mathrm{mol} / \mathrm{L}$ NAA were 2-5-fold higher than in calli exposed to the other NAA concentrations. This finding suggested that MdARF13 may repress anthocyanin biosynthesis.

\section{Bioinformatics analysis of the nuclear protein MdARF13}

Extensive bioinformatics and biochemical analyses of plants have clarified the number of $A R F$ genes and their potential functions. In $A$. thaliana, experiments have revealed that AtARF4 contains an active RD and functions as a repressor in plant protoplast transfection assays ${ }^{35}$. A phylogenetic analysis to elucidate the relationship between MdARF13 and ARF proteins from other plant species indicated that MdARF13 and AtARF4 belong to the same clade (Fig. 2a). Additionally, the aligned protein sequences revealed that MdARF13 and ARF proteins from other plant species contain a conserved B3-like DBD, an RD, and a domain homologous to the Aux/IAA proteins (CTD) (Fig. 2b).
The subcellular localization of MdARF13 was studied by introducing a 35S::MdARF13-GFP construct into the protoplasts of red-fleshed apple calli. The transgenic calli carrying the empty vector (35S::GFP) were used as the control. In protoplasts expressing 35S::MdARF13-GFP, the GFP signal was observed only in the nucleus, while the GFP signal was detected throughout the control protoplasts (Fig. 2c), implying that MdARF13 is localized to the nucleus.

\section{MdARF13 interacts with MdIAA121}

The ARFs contain a dimerization domain responsible for the formation of heterodimers with Aux/IAA proteins ${ }^{31}$. The $\mathrm{Y} 2 \mathrm{H}$ assays, which were used to test whether MdARF13 interacts with Aux/IAA proteins, revealed that MdARF13 interacts only with MdIAA121 (Fig. 3a). The BiFC assay involving onion epidermal cells cotransformed with MdARF13-NYFP and MdIAA121-CYFP constructs confirmed that MdARF13 interacts with MdIAA121 (Fig. 3b). The nuclei of these onion epidermal cells produced a strong YFP signal according to laser scanning confocal microscopy. In contrast, the YFP signal was not detected in cells in which MdIAA121-CYFP was replaced with CYFP alone. Meanwhile, our pull-down assays revealed that the recombinant MdIAA121-HIS fusion protein could be purified with MdARF13-GST, but not with GST alone (Fig. 3c). These results were consistent with those of the $\mathrm{Y} 2 \mathrm{H}$ and BiFC assays. Our data suggested that MdARF13 can interact with MdIAA121 under in vivo and in vitro conditions.

\section{Auxin affects MdIAA121 stability}

Ubiquitination, which represents an important posttranslational modification, is a key regulator of various processes, such as hormone signaling, responses to light and sugars, plant development, and plant immunity. In the Aux/IAA-ARF signaling pathway, auxin treatments promote the degradation of Aux/IAA by the $26 \mathrm{~S}$ proteasome to release ARF proteins ${ }^{46}$. We conducted protein degradation assays to study the posttranslational regulation of MdIAA121 in vitro. The MdIAA121-His proteins were rapidly degraded after NAA was added (Fig. 4a). Furthermore, samples were treated with MG132 and dimethyl sulfoxide (DMSO). The MG132 treatment noticeably weakened the effect of auxin on MdIAA121 stability (Fig. 4b). These findings indicated that auxin induces the degradation of MdIAA121 via the $26 \mathrm{~S}$ proteasome.

\section{Overexpression of MdARF13 repressed anthocyanin accumulation in the apple callus}

To examine whether MdARF13 affects anthocyanin accumulation, MdARF13 was overexpressed in redfleshed apple calli. The color of the resulting transgenic calli changed from dark red to yellow, while the transcript levels of anthocyanin biosynthesis-related genes and the accumulation of anthocyanin decreased (Fig. 5). These 


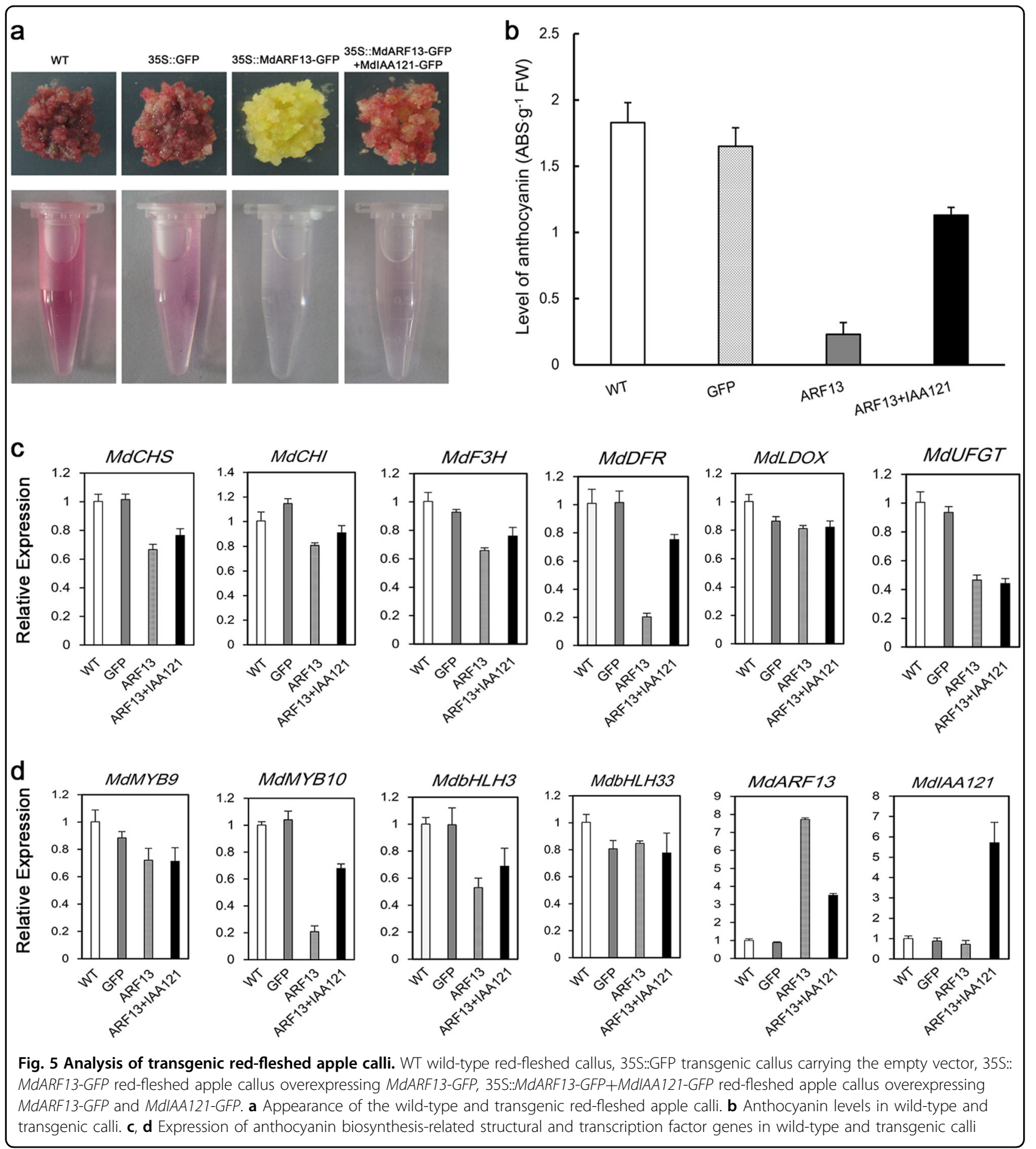

observations implied that MdARF13 represses anthocyanin biosynthesis. To assess whether MdIAA121 inhibits MdARF13 activities, we generated calli cotransfected with $M d A R F 13+M d I A A 121$ by $A$. tumefaciens-mediated transformation. The expression levels of anthocyanin structural genes and anthocyanin content were obviously higher in cotransfected calli than in calli overexpressing
MdARF13 (Fig. 5). These results demonstrated that MdARF13 together with MdIAA121 mediate auxin signaling to regulate anthocyanin biosynthesis.

\section{MdARF13 interacts with MdMYB10}

The MYB TFs are crucial for anthocyanin biosynthesis, and the expression of the corresponding genes is regulated 


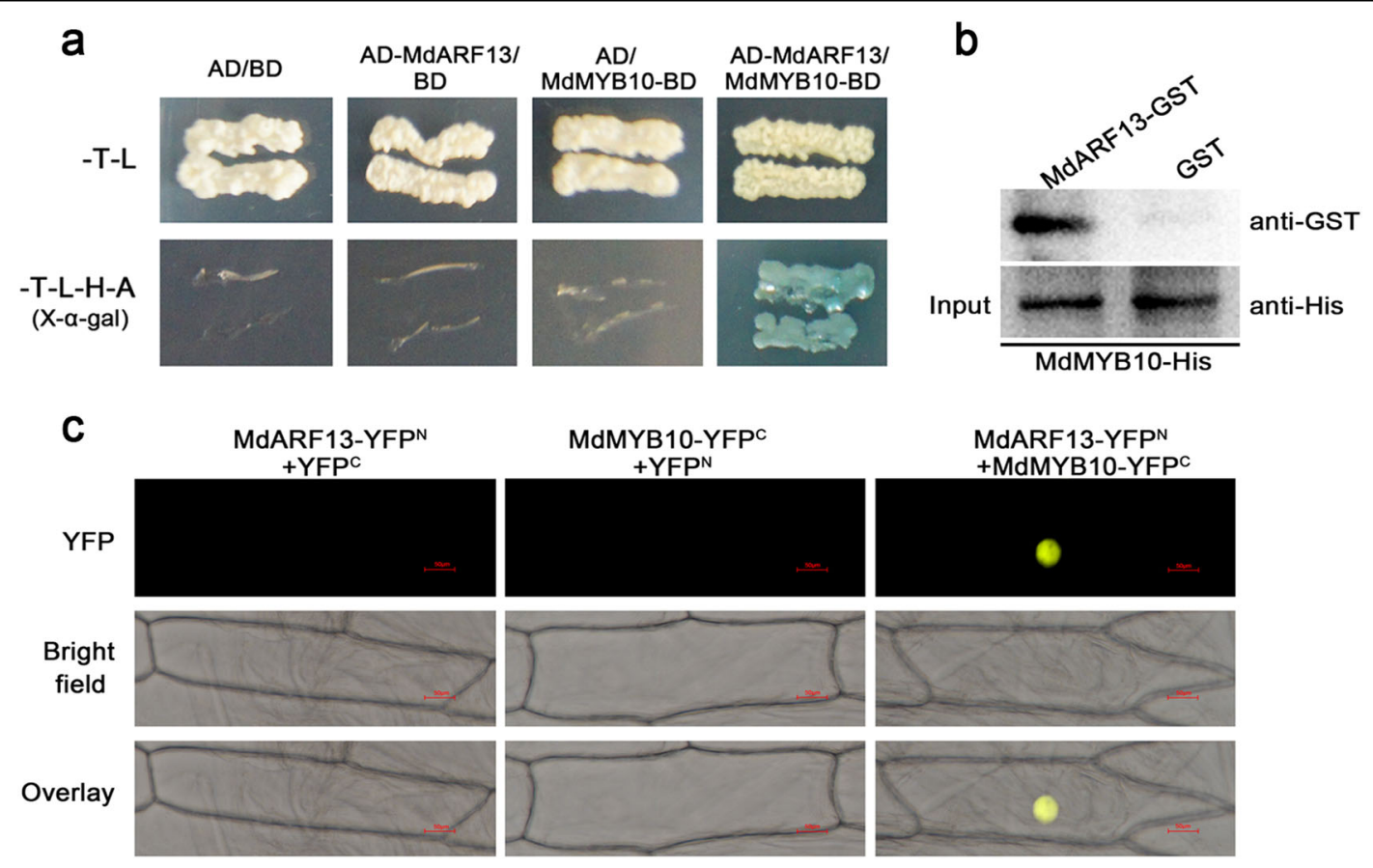

Fig. 6 Interaction between MdARF13 and MdMYB10. The interaction between MdARF13 and MdMYB10 was verified in a yeast two-hybrid, $\mathbf{b}$ pulldown, and $\mathbf{c}$ bimolecular fluorescence complementation assays

by the external environment. In this study, we hypothesized that MdARF13 is responsive to auxin and affects $M Y B$ expression. To verify this hypothesis, we completed $\mathrm{Y} 2 \mathrm{H}$ assays to investigate whether MdARF13 interacts with MdMYB9 and MdMYB10. We observed that MdARF13 interacts only with MdMYB10 (Fig. 6a). The physical interaction between MdARF13 and MdMYB10 was further confirmed in pull-down and BiFC assays (Fig. 6b, c). Thus, we speculated that interactions between MdARF13 and MdMYB10 affect anthocyanin biosynthesis.

\section{MdARF13 binds to the MdDFR promoter}

ARF TFs regulate their downstream target genes by binding to the TGTCTC AuxRE in the promoter region and subsequently promoting or repressing expression ${ }^{29}$. We analyzed the promoter elements of the anthocyanin biosynthesis structural genes (MdCHS, MdCHI, MdDFR, $M d L D O X, M d F L S$, and MdUFGT) using the PlantCARE cisacting regulatory element database (http://bioinformatics. psb.ugent.be/webtools/plantcare/html/) and determined that only MdDFR contains a putative AuxRE in its promoter region (Fig. 7b). We conducted an EMSA to clarify whether MdARF13 is recruited to the MdDFR promoter region. The assay results indicated that MdARF13 could interact with DNA probes containing the TGTCTC AuxRE (Fig. 7b). Moreover, $\mathrm{Y} 1 \mathrm{H}$ assays confirmed that MdARF13 could interact with the $M d D F R$ promoter (Fig. 7a), suggesting that MdARF13 suppresses the expression of MdDFR to decrease anthocyanin accumulation. Additionally, the ChIP-qPCR assay results suggested that the MdARF13-GFP fusion protein could bind to the DFR promoter, while the overexpression of MdIAA121 in MdARF13-overexpressing calli clearly inhibited the recruitment of MdARF13 to the $M d D F R$ promoter (Fig. 7c).

\section{Discussion}

Auxin has long been recognized for its essential role in plant growth and development. Previous studies concluded that auxin signaling regulates anthocyanin biosynthesis in plant calli, which is associated with changes in the external auxin concentration ${ }^{20,26}$. In separate studies, Ji et al. ${ }^{26}$ and Liu et $\mathrm{al}^{47}$ observed that increasing auxin concentrations within a certain range can inhibit anthocyanin biosynthesis in apple and $A$. thaliana. In this study, we observed that increasing NAA concentrations increased the inhibition of anthocyanin accumulation. Deikman and Hammer ${ }^{48}$ reported that the application of exogenous auxins suppressed the expression of anthocyanin regulatory and structural genes to varying degrees. Similarly, we observed that the expression levels of most structural and regulatory genes decreased in calli treated with NAA. Furthermore, the expression of genes encoding two types of TFs (Aux/IAA and ARF) associated with auxin signaling also increased to varying degrees, especially MdARF13. Thus, we speculate that these auxin signaling factors help regulate the expression of genes related to the anthocyanin biosynthesis pathway. 


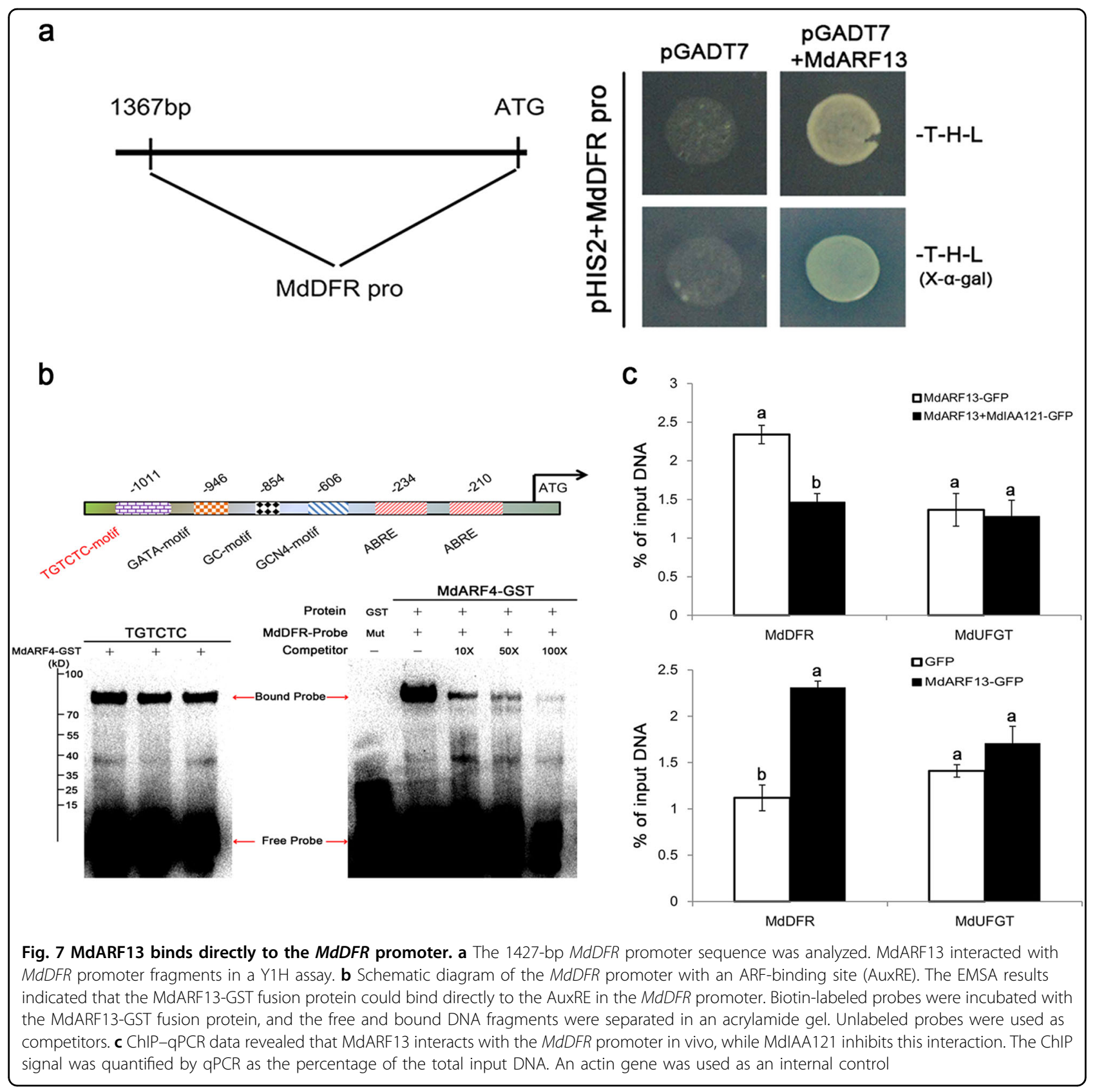

The Aux/IAA and ARF TFs are an integral part of the complex and elaborate auxin signaling pathway. Genomewide analyses of ARF families have been completed in many plant species, including rice, tomato, and maize ${ }^{49-51}$. In A. thaliana, in addition to ARF3, 13, 17, and 23, the ARF family mainly comprises proteins with the following three parts: an N-terminal DBD, a middle region that functions as an $\mathrm{AD}$ or $\mathrm{RD}$, and $\mathrm{CTD}^{29}$. In this study, we determined that MdARF13 is homologous to AtARF4 and has a similar structure. ARFs exist in the nucleus for a relatively short period ${ }^{29}$. Consistent with this previous report, we observed that MdARF13 is a nuclear protein. Most Aux/IAAs function as repressors and are believed to dimerize with ARF activators via their CTDs. However, the Aux/IAA repressors are rapidly degraded as auxin concentrations increase, thereby impairing their inhibitory effect on early auxin-responsive genes ${ }^{35}$. Our $\mathrm{Y} 2 \mathrm{H}, \mathrm{BiFC}$, and pull-down assays revealed that MdARF13 can interact with MdIAA121. Additionally, exogenous auxin treatment induces the degradation of MdIAA121 by the $26 \mathrm{~S}$ proteasome, suggesting that MdIAA121 might serve as a labile repressor of MdARF13. However, future studies will need to identify the specific ubiquitination mechanisms associated with MdIAA121 degradation. 


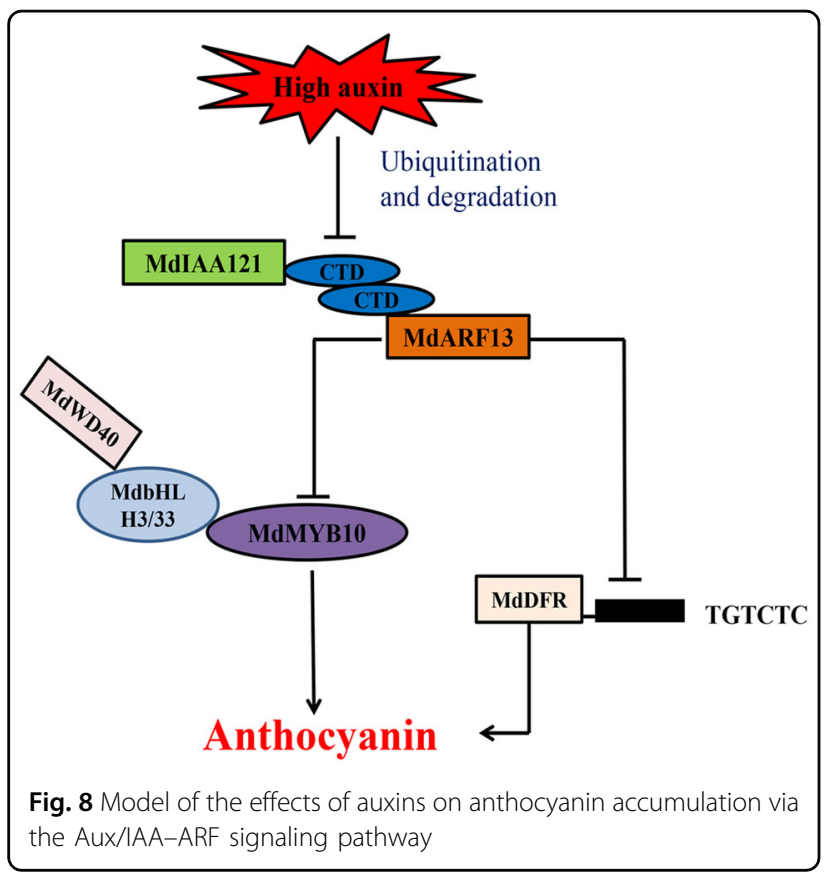

To further investigate the functions of MdARF13 during anthocyanin biosynthesis, MdARF13 was overexpressed in red-fleshed apple calli. A previous study involving transfected protoplasts indicated that AtARF4 functions as a transcriptional repressor ${ }^{35}$. In the current study, qPCR data revealed that the transcript levels of auxin-regulated anthocyanin biosynthesis-related genes were down-regulated more in the calli with the 35S::MdARF13-GFP construct than in the wildtype control. Thus, MdARF13 is likely an inhibitor of anthocyanin biosynthesis in apple. Additionally, Aux/IAA can function as a repressor that dimerizes with the target $\mathrm{ARF}^{52}$. We observed that overexpressing MdIAA121 in calli overexpressing $M d A R F 13$ weakened the inhibitory effect of MdARF13 on anthocyanin biosynthesis, suggesting that MdARF13 may be targeted by MdIAA121. The interaction between these two proteins may attenuate the inhibitory effect of MdARF13 on anthocyanin biosynthesis.

The MYB TFs are crucial for regulating anthocyanin biosynthesis in apples. External environmental factors affect anthocyanin accumulation because the associated signaling elements regulate the expression of $M Y B$ genes. An earlier study indicated that the regulation of anthocyanin biosynthesis by MdMYB1 is responsive to light, with COP1 functioning as a negative regulator that degrades MdMYB1 in darkness ${ }^{53}$. The HY5 TF targets the MYBD promoter in cytokinin-induced signaling pathways, leading to the accumulation of anthocyanin ${ }^{54}$. In this study, $\mathrm{Y} 2 \mathrm{H}, \mathrm{BiFC}$, and pull-down assays verified the in vitro and in vivo interaction between MdARF13 and MdMYB10, suggesting that MdARF13 might repress anthocyanin accumulation by down-regulating MdMYB10 expression. Moreover, ARF TFs contain DNA-binding motifs and play key roles in the regulation of downstream genes through TGTCTC sequence-specific interactions with promoters ${ }^{30}$. We detected an MdARF13-binding site upstream of the $M d D F R$ gene (Fig. 7a). EMSA, ChIP, and $\mathrm{Y} 1 \mathrm{H}$ assays were conducted to confirm the specific binding of MdARF13 to the MdDFR promoter. We also proved that MdIAA121 functions as a repressor of the auxin signal and interacts with MdARF13 to inhibit the recruitment of MdARF13 to the MdDFR promoter. Therefore, in apple, exogenous auxins repress anthocyanin biosynthesis most likely through the MdIAA121-MdARF13 signal transduction pathway.

ARF TFs are important for auxin signaling, and are involved in many processes related to plant growth and development. In this study, we observed that the inhibition of anthocyanin biosynthesis by high auxin concentrations involves MdARF13 (Fig. 8). Clarifying the role of auxin in the regulation of anthocyanin biosynthesis by ARFs may provide new insights into the regulation of anthocyanin metabolism by other hormones. During the cultivation of fruit trees, the rational application of hormones based on different environmental conditions can improve the appearance and nutritional value of fruit crops. Herein, we discussed the involvement of ARFs in the anthocyanin biosynthesis pathway, which may have implications for the development of new cultivation techniques aimed at improving fruit coloration under diverse environmental conditions.

\section{Acknowledgements}

This work was supported by grants from the National Key Research Project (2016YFC0501505) and the National Natural Science Foundation of China (31572091 and 31730080).

\section{Conflict of interest}

The authors declare that they have no conflict of interest.

\section{Publisher's note}

Springer Nature remains neutral with regard to jurisdictional claims in published maps and institutional affiliations.

Supplementary Information accompanies this paper at (https://doi.org/ 10.1038/s41438-018-0068-4).

Received: 17 March 2018 Revised: 7 June 2018 Accepted: 11 June 2018 Published online: 01 December 2018

\footnotetext{
References

1. King, M. C. \& Cliff, M. A. Development of a model for prediction of consumer liking from visual attributes of new and established apple cultivars. J. Am. Pom. Soc. 56, 223-229 (2002).

2. Horbowicz, M., Kosson, R., Grzesiuk, A. \& Debski, H. Anthocyanins of fruits and vegetables-their occurrence, analysis and role in human nutrition. Veg. Crops Res. Bull. 68, 5-22 (2008)

3. Mol, J., Grotewold, E. \& Koes, R. How genes paint flowers and seeds. Trends Plant Sci. 3, 212-217 (1998).

4. Knekt, P. et al. Dietary flavonoids and the risk of lung cancer and other malignant neoplasms. Am. J. Epidemiol. 146, 223-230 (1997).
} 
5. Middleton, J. E., Kandaswami, C. \& Theoharides, T. C. The effects of plant flavonoids on mammalian cells: implications for inflammation, heart disease, and cancer. Pharmacol. Rev. 52, 673-751 (2000).

6. Hollman, P. \& Katan, M. Absorption, metabolism and health effects of dietary flavonoids in man. Biomed. Pharmacother. 51, 305-310 (1997).

7. Zafra-Stone, S. et al. Berry anthocyanins as novel antioxidants in human health and disease prevention. Mol. Nutr. Food Res. 51, 675-683 (2007).

8. Baudry, A. et al. TT2, TT8, and TTG1 synergistically specify the expression of BANYULS and proanthocyanidin biosynthesis in Arabidopsis thaliana. Plant J. 39, 366-380 (2004).

9. Quattrocchio, F. et al. PH4 of petunia is an R2R3 MYB protein that activates vacuolar acidification through interactions with basic-helix-loop-helix transcription factors of the anthocyanin pathway. Plant Cell 18, 1274-1291 (2006).

10. Borevitz, J. O., Xia, Y., Blount, J., Dixon, R. A. \& Lamb, C. Activation tagging identifies a conserved MYB regulator of phenylpropanoid biosynthesis. Plant Cell 12, 2383-2394 (2000).

11. Cone, K. C., Cocciolone, S. M., Burr, F. A. \& Burr, B. Maize anthocyanin regulatory gene $\mathrm{pl}$ is a duplicate of $\mathrm{C} 1$ that functions in the plant. Plant Cell 5, 1795-1805 (1993).

12. Aharoni, A. et al. The strawberry FaMYB1 transcription factor suppresses anthocyanin and flavonol accumulation in transgenic tobacco. Plant J. $\mathbf{2 8}$ 319-332 (2001).

13. Deluc, L. et al. The transcription factor $V_{V M Y B 5 b}$ contributes to the regulation of anthocyanin and proanthocyanidin biosynthesis in developing grape berries. Plant Physiol. 147, 2041-2053 (2008).

14. Mcghie, T. K. et al. An R2R3 MYB transcription factor associated with regulation of the anthocyanin biosynthetic pathway in rosaceae. BMC Plant Biol. 10, 50 (2010).

15. Takos, A. M. et al. Light-induced expression of a MYB gene regulates anthocyanin biosynthesis in red apples. Plant Physiol. 142, 1216-1232 (2006).

16. Ban, Y. et al. Isolation and functional analysis of a MYB transcription factor gene that is a key regulator for the development of red coloration in apple skin. Plant Cell Physiol. 48, 958-970 (2007).

17. Espley, R. V. et al. Red colouration in apple fruit is due to the activity of the MYB transcription factor, MdMYB10. Plant J. 49, 414-427 (2007).

18. Cominelli, E. et al. Expression analysis of anthocyanin regulatory genes in response to different light qualities in Arabidopsis thaliana. J. Plant Physiol. 165, 886-894 (2008)

19. Rowan, D. D. et al. Environmental regulation of leaf colour in red 35S:PAP1 Arabidopsis thaliana. New Phytol. 182, 102-115 (2009).

20. Ozeki, Y. \& Komamine, A. Effects of growth regulators on the induction of anthocyanin synthesis in carrot suspension cultures. Plant Cell Physiol. 27, 1361-1368 (1986).

21. Qi, T. et al. The Jasmonate-ZIM-Domain proteins interact with the WD-Repeat/ $\mathrm{bHLH/MYB}$ complexes to regulate jasmonate-mediated anthocyanin accumulation and trichome initiation in Arabidopsis thaliana. Plant Cell $\mathbf{2 3}$ 1795-1814 (2011)

22. Meyer, H. J. \& Staden, J. The in vitro production of an anthocyanin from callus cultures of Oxalis linearis. Plant Cell Tissue Organ Cult. 40, 55-58 (1995).

23. Ozeki, Y. \& Komamine, A. Effects of inoculum density, zeatin and sucrose on anthocyanin accumulation in a carrot suspension culture. Plant Cell Tissue Organ Cult. 5, 45-53 (1985).

24. Zhou, L. L., Zeng, H. N., Shi, M. Z. \& Xie, D. Y. Development of tobacco callus cultures over expressing Arabidopsis PAP1/MYB75 transcription factor and characterization of anthocyanin biosynthesis. Planta 229, 37-51 (2008).

25. Murthy, H. N., Lee, E. J. \& Paek, K. Y. Production of secondary metabolites from cell and organ cultures: strategies and approaches for biomass improvement and metabolite accumulation. Plant Cell Tissue Organ Cult. 118, 1-16 (2004).

26. Ji, X. H. et al. Effect of auxin, cytokinin and nitrogen on anthocyanin biosynthesis in callus cultures of red-fleshed apple (Malus sieversii f. niedzwetzkyana). Plant Cell Tissue Organ Cult. 120, 325-337 (2015).

27. Ji, X. H., Zhang, R., Wang, N., Yang, L. \& Chen, X. S. Transcriptome profiling reveals auxin suppressed anthocyanin biosynthesis in red-fleshed apple callus (Malus sieversii f. niedzwetzkyana). Plant Cell Tissue Organ Cult. 123, 389-404 (2015).

28. Guilfoyle, T. J., Ulmasov, T. \& Hagen, G. The ARF family of transcription factors and their role in plant hormone-responsive transcription. Cell Mol. Life Sci. 54, 619-627 (1998).
29. Ulmasov, T., Hagen, G. \& Guilfoyle, T. J. Activation and repression of transcription by auxin-response factors. Proc. Natl. Acad. Sci. USA 96, 5844-5849 (1999).

30. Tiwari, S. B., Wang, X. J., Hagen, G. \& Guilfoyle, T. J. AUX/IAA proteins are active repressors, and their stability and activity are modulated by auxin. Plant Cell $\mathbf{1 3}$, 2809-2822 (2001).

31. Guilfoyle, T. J. \& Hagen, G. Auxin response factors. J. Plant Growth Regul. 10, 281-291 (2001).

32. Liscum, E. \& Reed, J. W. Genetics of Aux/IAA and ARF action in plant growth and development. Plant Mol. Biol. 49, 387-400 (2002).

33. Benfey, P. N. Auxin action: slogging out of the swamp. Curr. Biol. 12 R389-R390 (2002).

34. Hellmann, H. \& Estelle, M. Plant development: regulation by protein degradation. Science 297, 793-797 (2002).

35. Tiwari, S. B., Hagen, G. \& Guilfoyle, T. The roles of auxin response factor domains in auxin-responsive transcription. Plant Cell 15, 533-543 (2003).

36. Sessions, A. et al. EITIN patterns the Arabidopsis floral meristem and reproductive organs. Development 124, 4481-4491 (1997).

37. Pekker, I., Alvarez, J. P. \& Eshed, Y. Auxin response factors mediate Arabidopsis organ asymmetry via modulation of KANADI activity. Plant Cell 17, 2899-2910 (2005).

38. Hardtke, C. S. et al. Overlapping and nonredundant functions of the Arabidopsis auxin response factors MONOPTEROS and NONPHOTOTROPIC HYPOCOTYL 4. Development 131, 1089-1100 (2004).

39. Hardtke, C. S. \& Berleth, T. The Arabidopsis gene MONOPTEROS encodes a transcription factor mediating embryo axis formation and vascular development. EMBO J. 17, 1405-1411 (1998).

40. Tian, C. E. et al. Disruption and overexpression of auxin response factor 8 gene of Arabidopsis affect hypocotyl elongation and root growth habit, indicating its possible involvement in auxin homeostasis in light condition. Plant J. 40, 333-343 (2004).

41. Vriezen, W. H., Feron, R., Maretto, F., Keijman, J. \& Mariani, C. Changes in tomato ovary transcriptome demonstrate complex hormonal regulation of fruit set. New Phytol. 177, 60-76 (2008).

42. De, J. M., Woltersarts, M., Feron, R., Mariani, C. \& Vriezen, W. H. The Solanum lycopersicum auxin response factor 7 (SIARF7) regulates auxin signaling during tomato fruit set and development. Plant J. 57, 160-170 (2009).

43. Sun, J. et al. Effects of methyl jasmonate and abscisic acid on anthocyanin biosynthesis in callus cultures of red-fleshed apple (Malus sieversii, f. niedzwetzkyana). Plant Cell Tissue Organ Cult. 130, 227-237 (2017).

44. An, X. H. et al. MdMYB9 and MdMYB11 are involved in the regulation of the JA-induced biosynthesis of anthocyanin and proanthocyanidin in apples. Plant Cell Physiol. 56, 650-662 (2015).

45. Hu, D. G. et al. MdMYB1 regulates anthocyanin and malate accumulation by directly facilitating their transport into vacuoles in apples. Plant Physiol. 170, 1315-1330 (2016).

46. Smalle, J. \& Vierstra, R. D. The ubiquitin 265 proteasome proteolytic pathway. Annu. Rev. Plant Biol. 55, 555-590 (2004).

47. Liu, Z., Shi, M. Z. \& Xie, D. Y. Regulation of anthocyanin biosynthesis in arabidopsis thaliana red pap1-d cells metabolically programmed by auxins. Planta 239, 765-781 (2014).

48. Deikman, J. \& Hammer, P. E. Induction of anthocyanin accumulation by cytokinins in Arabidopsis thaliana. Plant Physiol. 108, 47-57 (1995).

49. Wang, D. et al. Genomewide analysis of the auxin response factors (ARF) gene family in rice (Oryza sativa). Gene 394, 13-24 (2007).

50. Kumar, R., Tyagi, A. K. \& Sharma, A. K. Genome-wide analysis of auxin response factor (ARF) gene family from tomato and analysis of their role in flower and fruit development. Mol. Genet. Genomics 285, 245-260 (2011).

51. Xing, H. et al. Genome-wide identification and expression profiling of auxin response factor (ARF) gene family in maize. BMC Genomics 12, 178 (2011).

52. Tiwari, S. B., Hagen, G. \& Guilfoyle, T. J. Aux/iaa proteins contain a potent transcriptional repression domain. Plant Cell 16, 533-543 (2004).

53. Li, Y. Y. et al. Mdcop1 ubiquitin e3 ligases interact with mdmyb1 to regulate light-induced anthocyanin biosynthesis and red fruit coloration in apple. Plant Physiol. 160, 1011-1022 (2012).

54. Nguyen, N. H. et al. MYBD employed by HY5 increases anthocyanin accumulation via repression of MYBL2 in Arabidopsis. Plant J. 84, 1192-1205 (2015). 